\title{
The Prevalence of dysglycaemia and other cardiovascular risk factors among first degree relatives of Irish patients with type 2 diabetes in Ireland
}

\begin{abstract}
Objectives: To assess the prevalence of diabetes, pre-diabetes and other cardiovascular risk factors amongst close relatives of patients with type 2 diabetes.

Methods: 100 participants aged between 25 and 75 years were recruited through their first degree relatives, who have type 2diabetes and attend Naas Hospital diabetes clinic. Participants underwent an oral glucose tolerance test, cardiovascular risk factor assessment and completed a questionnaire.

Results: $60 \%$ of participants were females with a mean age of 45.9 years, $95 \%$ CI (44.08-47.8). 21\% had dysglycaemia (One with diabetes and 20 pre-diabetes). $34 \%$ had un-diagnosed hypertension and $8 \%$ were known hypertensive but not controlled. $71 \%$ had dyslipidaemia. $23 \%$ were overweight, while $38 \%$ were obese. Fasting blood glucose correlated positively with age $(p=0.047)$, BMI $(p=0.02)$, systolic BP $(p=0.003)$ and triglycerides levels $(\mathrm{p}=0.023)$.

Conclusion: First degree relatives of type 2 diabetes patients showed relatively low prevalence of dysglycaemia, but a high prevalence of dyslipidaemia, hypertension and obesity.
\end{abstract}

Volume 7 Issue 3 - 2019

\section{Fedail M, Hajer E, Moore K}

Department of Diabetes and Endocrinology, Naas General Hospital, Europe

Correspondence: Fedail M, Department of Diabetes and Endocrinology, Naas General Hospital, Europe,

Email moawiafedail200I@yahoo.com

Received: March 12, 2019| Published: May 24, 2019

\section{Introduction}

It is well known that a considerable number of people with type 2 diabetes are diagnosed late and by the time they are diagnosed, they could have already developed microvascular or macrovascular complications of diabetes. Early detection and management of cardiovascular risk factors including diabetes might improve the outcome. High concordance rates for type 2 diabetes in identical twins, ${ }^{1,2}$ the high prevalence of diabetes in people from certain families $^{3,4}$ and races, ${ }^{5,6}$ all have supported the presence of genetic determinants for type 2 diabetes. In spite of the presence of many genetic variants that are linked to the risk of developing diabetes, their clinical usefulness to estimate the risk for diabetes in the population is limited. ${ }^{7,8}$ Well established risk factors for type 2 diabetes include age, sex, obesity physical inactivity, dyslipidemia and family history. Studies in U.S. population have shown a graded and independent role for the family history as a risk for developing type 2 diabetes. ${ }^{10,11}$ First degree relatives of patients with type 2 diabetes are at a higher risk of developing type 2 diabetes, not only because of their genetic predisposition but also due to their life style habits in relation to diet. ${ }^{12} \mathrm{~A}$ Chinese study assessing the impact of a positive family history of type 2 diabetes on the B-cell function of close relatives has demonstrated decline in B-cell function compared to those without family history of diabetes. However, there was no difference in the insulin resistance between the study groups. ${ }^{13}$ This supports that some first degree relatives of people with diabetes are subject to a continuous process of metabolic dysfunction and hyperglycemia that pass through stages and end in type 2 diabetes. The overall prevalence of diabetes in Ireland was estimated to be $6.1 \%$ in 2005 , type 2 diabetes accounted for $90 \%$ of cases. Prevalence in females was higher $5.4 \%$ versus $4.0 \%$ for males. Moreover, the prevalence in people over 60 years was even higher $13.8 \%{ }^{14}$ The Irish college of General practitioners (ICGP) in collaboration with the Irish Endocrine Society (IES) and Health Service Executives (HSE) have issued guidelines with regard to screening for type 2 diabetes in July $2008 .{ }^{15}$ In a first randomized controlled trial, Researchers from Cambridge, have recently found that, screening for T2 DM doesn't reduce mortality at $10 \mathrm{yrs}^{16}$

This contradicts evidence from previous studies. ${ }^{17}$ The lack of difference in mortality between the studied groups has been explained by several factors including the dilution effect of screening and the improvement in detection and management of cardiovascular disease risk factors including diabetes in UK primary care enhanced by the Quality and Outcomes Frame work system of remuneration for general practioners. ${ }^{18}$ This might have contributed to a significantly lower mortality than expected in the high risk individuals in the control group, according to observational studies in Netherland. ${ }^{19}$ No previous studies have looked at the prevalence of type 2diabetes, pre-diabetes and other cardiovascular risk factors in the first degree relatives of Irish patients with T2DM. The primary objective of this study was to assess the prevalence of type 2 diabetes a pre-diabetes and other cardiovascular risk factors in an Irish cohort of first degree relatives of patients with type 2 diabetes.

\section{Methods}

\section{Design}

This study was based on a cross sectional survey. Close relatives (Brothers, Sisters, and Parents under 75 years of age and children over 30 years) of patients with type 2 diabetes were screened for diabetes pre-diabetes (impaired fasting glycemia and impaired glucose tolerance) and other cardiovascular risk factors. 


\section{Sample}

The participants were recruited through their close relatives who were attending our weekly type 2 diabetes clinic in the hospital. A letter describing the study (the importance of the screening for this group of people, what is involved in this screening, possible results and how different results will be dealt with) was handed out to the patients attending our diabetes clinic on weekly base. This step was followed by a phone call to these patients a week later to get their close relatives contact details for direct invitation for screening if they are happy to proceed. The size of the sample to be studied was designed to be around 100 participants.

The inclusion criteria: Close relatives of patients with type 2 diabetes, males or females including siblings and children aged thirty years and up to seventy four years.

Exclusion criteria: Non first degree relatives .Age less than 30 years or greater than 74 years, people on steroids, pregnant ladies, people with mental illness, and terminally ill people, People with dementia or learning disabilities, people known to have diabetes. People who had a diabetes screening test less than one year.

\section{Materials}

The data were collected through a questionnaire which was filled out during an interview, blood samples for fasting blood glucose, lipid profile, $\mathrm{Hb} \mathrm{A} 1 \mathrm{c}$ and 2 hours post prandial blood glucose were taken. Blood pressure was checked and body mass index (BMI) measured (using a height and weight measuring scales) as other risk factors for diabetes needed to be ascertained.

\section{Procedure}

Participants were recruited through their first degree relatives. An information letter detailing the purpose of the study and the benefits from participation was distributed to the patients while a waiting to be seen in the diabetes clinic. First degree relatives of patients with type 2 diabetes who gave permission to be contacted and they were willing to participate, were contacted by phone and invited to participate through attendance to the hospital fasting from midnight the day before their tests. The tests carried-out involved, fasting blood sugar, oral glucose tolerance test, and lipids profile. The ADA diagnostic criteria (2003) were used to interpret the results. ${ }^{20}$

\section{Data analysis}

The data was analyzed using SPSS version 18.0.Statistical methods used included independent t-test and spearman rank correlations. All tests for statistical significance were 2 tailed. The level of significance for $\mathrm{P}$ value was $<0.05$.

\section{Results}

$60 \%$ of the participants were females; median age was 46.5 , range (26- 68 years). $21 \%$ had dysglycemia; one participant had diabetes and 20 pre-diabetes (Table 1). 34\% had undiagnosed hypertension and $8 \%$ were known hypertensive but uncontrolled. $71 \%$ of participants had dyslipidaemia; $59 \%$ had high total cholesterol, $27.5 \%$ had high triglyceride and $58 \%$ had high LDL. $40 \%$ of participants were overweight with a BMI $>25$ and a further $38 \%$ were obese (Table 2). Fasting blood glucose (FBG) correlated positively with age $(\mathrm{p}=0.047)$, BMI $(\mathrm{p}=0.02)$, systolic $\mathrm{BP}(\mathrm{p}=0.003)$ and triglycerides $(\mathrm{p}=0.023)$. FBG correlated negatively with HDL $(\mathrm{p}=0.005) .2$ hour post prandial glucose correlated positively with BMI $(\mathrm{p}=0.04)$ and triglyceride level $(\mathrm{p}=0.028)$, but did not correlate with HDL (Figure 1). The level of education achieved by participants (secondary or third level) and quantity of physical activity undertaken per week did not correlate with fasting or postprandial blood glucose level. $23.7 \%$ of participants had previously been screened for diabetes, of which only 14 had been screened in the previous 3 years. Moreover, $43.8 \%$ did not know that they were at increased risk of developing diabetes.

Table I Means of Impaired Fasting Glycaemia (IFG) and Impaired Glucose Tolerance (IGT) for males and females

\begin{tabular}{lll}
\hline Gender & $\begin{array}{l}\text { Mean FBG } \\
(\mathbf{m m o l} / \mathbf{l})\end{array}$ & $\begin{array}{l}\text { Mean } 2 \mathrm{Hrs} \text { PP } \\
\text { glucose( } \mathbf{m m o l} / \mathbf{L})\end{array}$ \\
\hline Males & $4.9 \pm 0.57$ & $5.3 \pm 1.59$ \\
Females & $5.06 \pm 1.1$ & $5.75 \pm 2.1$ \\
\hline
\end{tabular}

Table 2 The distribution of dysglycemia by body mass Index (BMI)

\begin{tabular}{ll}
\hline BMI & $\begin{array}{l}\text { Percentage of individuals } \\
\text { with dysglycemia }\end{array}$ \\
\hline Less than 25 & 19.1 \\
Greater than 25 & 80.9
\end{tabular}

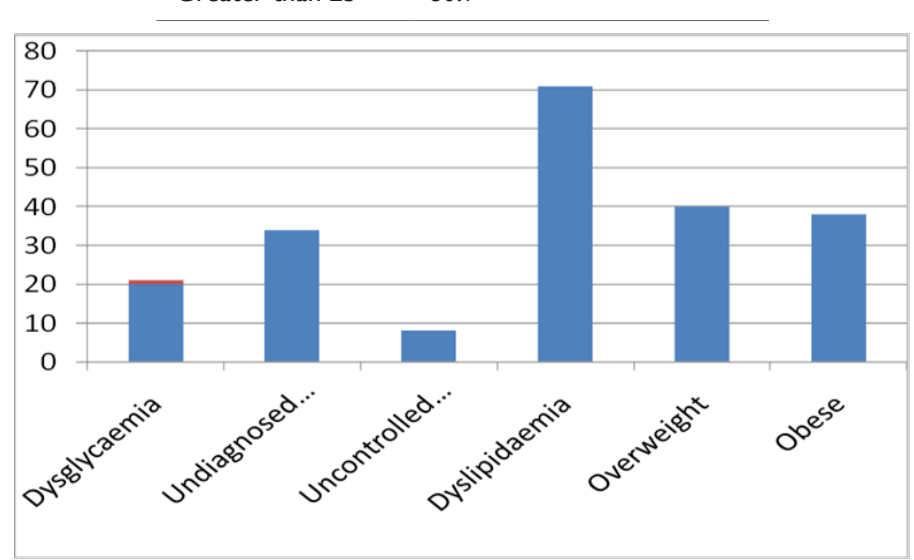

Figure I Prevalence of cardiovascular risk factors (\%).

\section{Discussion}

Abnormal glucose metabolism is common amongst adult relatives of patients with type 2 diabetes. Studies have shown that people who have one first degree relative (FDR) suffering from diabetes have a $40 \%$ risk of having this disease and if diabetes is present in both parents, this risk is almost doubled..$^{21}$ In this cross-sectional study, FDR of patients with diabetes, have a relatively low prevalence of diabetes but a high prevalence of pre-diabetes. Differences in sample sizes, the characteristics of studied populations and the strength of genetic factors might have contributed. The prevalence of cardiovascular risk factor abnormalities in our population is alarmingly high. The prevalence of hypertension in our cohort was $42.8 \%$. Data from the Irish Institute of Public Health (IPH) showed that the prevalence of high BP among the general population in 2007, was $25.1 \%$. This high prevalence compared to the general population is expected as the participants in our cohort were high risk group of population, being first degree relatives of patients with type 2 diabetes and with high prevalence of obesity and dyslipidaemia. The increased prevalence of high triglycerides and low high density lipoprotein levels (HDL)in 
this cohort of first degree relatives is consistent with the findings in a previous study from small town in Argentina with high prevalence of type 2 diabetes, where family members of patients with an established diagnosis of type 2 diabetes mellitus who are theoretically at risk of having the metabolic syndrome, were found to have high prevalence of reduced levels of HDL and increased levels of triglycerides. ${ }^{22}$ The HDL and triglycerides abnormalities are most likely related to the abnormal glucose metabolism and insulin resistance.

Our results suggest that high risk individuals are unaware of their risk of diabetes, $43.8 \%$ of those who had their knowledge status documented did not know that they were at increased risk of developing diabetes. The first degree relatives in our study population are not receiving appropriate screening. Only $23(23.7 \%)$ of the participants were known to have previously been screened for diabetes, of whom only $14(60.9 \%)$ had been screened in the previous 3 years. The strengths in our study include the broader age groups of participants including both females and males and the ability to give information on the independent role of some other risk factors for impaired glucose metabolism. Like other studies, our study had some limitations which include; it's cross sectional nature which can only allow for causal relationships between risk factors and impaired glucose metabolism. Another limitation was the relatively small sample of the participants. However, despite these limitations, our findings were important and have added valuable information to our understanding to the prevalence of impaired glucose metabolism and more importantly, the other cardiovascular risk factors in this group of Irish population. Also we were able to demonstrate poor knowledge towards the risk of acquiring type 2 diabetes and screening practices which lags behind the recommendations. To conclude, this is the first Irish study to show the prevalence of diabetes, impaired glucose tolerance and impaired fasting glycaemia in addition to the other cardiovascular risk factors in the first degree relatives of Irish population with type 2 diabetes. The results support the importance of regular screening for diabetes, prediabetes and other cardiovascular risks in this population together with primary care led programmes, perhaps with media contribution to increase the awareness and encourage the uptake of regular screening for this at risk group in addition to running programmes to help with the prevention through lifestyle interventions.

\section{Acknowledgments}

None.

\section{Conflicts of interest}

The authors declare that there is no conflict of interest.

\section{References}

1. Eff C, Leslie D, Pyke D. Diabetes in identical twins: a study of 200 pairs Diabetologia. 1981;20(2):87-93.

2. Newman B, Selby JV, King MC, et al. Concordance for type 2 (noninsulin-dependent) diabetes mellitus in male twins. Diabetologia. 1987;30(10):763-768.

3. Harlan LC, Harlan WR, Landis JR, et al. Factors associated with glucose tolerance in adults in the United States. Am J Epidemiol. 1987;126:674684.

4. Morris RD, Rimm DL, Hartz AJ, et al. Obesity and heredity in the etiology of non-insulin-dependent diabetes mellitus in 32,662 adult white women. Am J Epidemiol. 1989;130(1):112-121.
5. Gardner LI, Stern MP, Haffner SM, et al. Prevalence of diabetes in Mexican Americans: relationship to percent of gene pool derived from Native American sources. Diabetes. 1984;33(1):86-92.

6. Lee ET, Howard BV, Savage PJ, et al. Diabetes and impaired glucose tolerance in three American Indian populations aged 45-74 years: the Strong Heart Study. Diabetes Care. 1995;18(5):599-610.

7. Weedon MN, McCarthy MI, Hitman G, et al. Combining information from common type 2 diabetes risk polymorphisms improves disease prediction. PLoS Med. 2006;3:e374

8. Siewert S, Filipuzzi S, Codazzi L, et al. Impact of Metabolic Syndrome Risk Factors in First-Degree Relatives of Type 2 Diabetic Patients. Rev Diabet Stud. 2007;4(3):177-184.

9. National Diabetes Information Clearinghouse. Am I at risk for type 2? Diabetes.

10. Annis AM, Caulder MS, Cook ML, et al. Family history, diabetes, and other demographic and risk factors among participants of the National Health and Nutrition Examination Survey 1999-2002. Prev Chronic Dis. 2005;2(2):A19.

11. Hariri S, Yoon PW, Qureshi N, et al. Family history of type 2 diabetes: a population-based screening tool for prevention? Genet Med. 2006;8(2):102-108

12. Adamson AJ, Foster E, Butler TJ, et al. Non-diabetic relatives of Type 2 diabetic families: dietary intake contributes to the increased risk of diabetes. Diabetic Medicine. 2001;18(12):984-990.

13. Wang ZH, Zhang SH, Gong LL, et al. The impact of family history of type 2 diabetes on pancreatic beta-cell function. Diabetes Research and Clinical Practice. 2009;86(1):61-66.

14. IPH. IPH predicts dramatic rise in chronic diseases - hypertension, coronary heart disease, stroke and diabetes (ROI data).

15. Department of Health and children. Irish college of General Practitioners and Irish Endocrine Society: A practical Guide to Integrated Type 2. Diabetes Care. 2008.

16. Simmons, Echouffo-Tcheugui JB, Sharp SJ, et al. Screening for type 2 Diabetes and population mortality over 10 years (ADDITIONCambridge): a cluster- randomized controlled trial. The Lancet. 2012;380(9855):1741-1748.

17. Griffin SJ, Borch-Johnsen K, Davies MJ, et al. Effect of early intensive multifactorial therapy on 5-year cardiovascular outcomes in individuals with type 2 diabetes detected by screening (ADDITION-Europe): a cluster-randomized trial. Lancet. 2011;378(9786):156-167.

18. Doran T, Fullwood C, Gravelle H, et al. Pay-for performance programs in family practices in the United Kingdom. NEngl JMed. 2006;355:375-384

19. Spijkerman A, Griffin S, Dekker J, et al. What is the risk of mortality for people who are screen positive in a diabetes screening programme but who do not have diabetes on biochemical testing? Diabetes screening programmes from a public health perspective. J Med Screen. 2002;9(4):187-190.

20. American Diabetes Association. Economic costs of diabetes in the U.S. in 2002. Diabetes Care. 2003;26(3):917-932.

21. Yuturu S, Bridges JF, Dhanireddy RR. Preliminary evidence of genetic anticipation in type 2 diabetes mellitus. Med Sci Monit. 2005;11(6):262-265.

22. Siewert S, Filipuzzi S, Codazzi L, et al. Impact of Metabolic Syndrome Risk Factors in First-Degree Relatives of Type 2 Diabetic Patients. Rev Diabet Stud. 2007;4(3):177-184. 